\title{
Efficacy of "Family Connections", a program for relatives of people with borderline personality disorder, in the Spanish population: study protocol for a randomized controlled trial
}

\author{
Isabel Fernández-Felipe ${ }^{1 *}$ (D), Verónica Guillén², Helio Marco², Amanda Díaz-García1, Cristina Botella',
} Mercedes Jorquera ${ }^{3}$, Rosa Baños ${ }^{2}$ and Azucena García-Palacios ${ }^{1}$

\begin{abstract}
Background: Patients with borderline personality disorder (BPD) experience significant affect regulation difficulties that cause serious consequences in their work, emotional, and social environments. This dysfunctional pattern also produces great suffering and a heavy burden on their relatives. Fortunately, some studies show that treatment of relatives of people with BPD begins to be important in the patients' recovery and in improving family dynamics. One of the treatments that has obtained the most empirical support is Family connections (FC). This 12-session program is an adaptation of different Dialectical Behavior Therapy strategies. To test the efficacy of FC, five uncontrolled clinical trials were conducted, with pre-post treatment and follow-up assessments. The results of these studies and subsequent replications showed an improvement in family attitudes and caregiver burnout. Our research team adapted FC for delivery in the Spanish population. We intend to test the efficacy of this program versus a treatment as usual condition. Moreover, we aim to test the efficacy of this program and study its effectiveness (in terms of participants' acceptance). This paper presents the study protocol.

Methods: The study is a randomized controlled trial. The participants will be recruited in a Personality Disorders Unit and randomly assigned to one of two treatment conditions: Family Connections group (FC) or Treatment As Usual (TAU). Primary outcome measures will be the BAS and FAD-GFS. Secondary outcomes will include DASS-21, FES, GS, and QLI. Participants' treatment acceptance and degree of satisfaction will also be measured. Participants will be assessed at pre-, post-treatment, and 6-month follow-up. Intention to treat and per protocol analyses will be performed.

* Correspondence: fernandi@uji.es

'Universitat Jaume I, Castellón, Spain

Full list of author information is available at the end of the article

C C The Author(s). 2020 Open Access This article is licensed under a Creative Commons Attribution 4.0 International License, which permits use, sharing, adaptation, distribution and reproduction in any medium or format, as long as you give appropriate credit to the original author(s) and the source, provide a link to the Creative Commons licence, and indicate if changes were made. The images or other third party material in this article are included in the article's Creative Commons licence, unless indicated otherwise in a credit line to the material. If material is not included in the article's Creative Commons licence and your intended use is not permitted by statutory regulation or exceeds the permitted use, you will need to obtain permission directly from the copyright holder. To view a copy of this licence, visit http://creativecommons.org/licenses/by/4.0/ The Creative Commons Public Domain Dedication waiver (http://creativecommons.org/publicdomain/zero/1.0/) applies to the data made available in this article, unless otherwise stated in a credit line to the data. 
(Continued from previous page)

Discussion: This is the first study on FC for relatives of people with borderline personality disorder (BPD) compared to an active condition (TAU), and this is the first time relatives' and patients' data will be analyzed. In addition, it is the first study to test the efficacy of the program in Spain. This intervention could contribute to improving the efficiency and effectiveness of current treatment programs for relatives of people with BPD, help to decrease burden, and improve the family connection.

Trial registration: ClinicalTrials.gov ID: NCT04160871. Registered November 15th 2019.

Keywords: Borderline personality disorder, Family connections, Relatives, DBT, Intervention, Caregivers, Burden

\section{Background}

Borderline personality disorder (BPD) is one of the most challenging and complex mental disorders. BPD is related to high suicide and self-harm rates. Persistent suicidal behavior is described in $69-80 \%$ of people with BPD [1]. A longitudinal study across 24 years comparing BPD and other personality disorders found that a total of $5.9 \%$ of BPD sufferers died by suicide and $14 \%$ by other causes, compared to 1.4 and $5.5 \%$ in a sample of people with other personality disorders [2]. BPD also involves high rates of hospital admissions and health service use. BPD is associated with a high economic burden due to the long-term use of health services [3-6], including interventions in emergency settings and the need for the services of several different professionals [6-8]. Furthermore, BPD is an important public mental health problem that produces great suffering for patients and their relatives [9]. For this reason, there is a need to provide specialized care.

The symptoms of BPD and their consequences lead to high levels of discomfort and burden for their relatives [10-14]. Additionally, there is evidence that maladaptive family communication patterns play a role in the etiology and maintenance of BPD [14, 15].

Family members of people with BPD are more likely to have psychological problems $[16,17]$, and they describe feelings of confusion, lack of awareness, and incompetence $[14,18,19]$. Studies with relatives of people with BPD showed that the levels of burden and depression can increase due to lack of clear knowledge about the diagnosis and the evolution of the disorder $[19,20]$. Moreover, when family members participate in treatment, patient relapse decreases, recovery is easier, and wellbeing in the family improves $[20,21]$.

Currently, there are interventions for family members with empirical support. All these programs are offered in group format, but they differ in the type of orientation and contents. So far, two of these studies present only psychoeducational contents; one is based on mentalization [22], and the other combines cognitive analytical therapy with general psychiatric care [23]. Regarding the programs that offer skills training, almost all are DBTbased programs or DBT adaptations. These DBT skills training studies have different structures and numbers of sessions. They use either adaptations of DBT in 10-12 sessions where parents receive training in DBT miniskills [17, 24-27] or group therapy where skills are taught for 6 months [28].

Family Connections (FC) is the most empirically supported program [25] for relatives of patients with BPD. The program can be carried out by clinicians or trained relatives. To date, five uncontrolled clinical trials have been performed with pre- and post-treatment and follow-up assessments $[17,24,25,29,30]$. In all the replications, the results of the FC program were consistent, showing significant decreases in burden, grief, anxiety, and depression, and significant increases in the participants' subjective experience of mastery, empowerment, well-being variables, and family functioning. Furthermore, these variations were maintained or improved at 3- or 6-month follow up. The good results for family functioning could be due to the fact that FC validates patients' skillful behaviors, decreases their psychological symptoms, improves interpersonal relationships between family members and patients, increases understanding of the problem, reduces perceived stigma, and enhances family empowerment [30].

$\mathrm{FC}$ is a program for relatives of people with BPD that was developed within the National Education Alliance for Borderline Personality Disorder [17, 25]. This program links three important needs for relatives: first, education about the disorder and family functioning; second, individual and family skills to manage negative reactions in the family and improve well-being in the relationship; and, finally, social support from other relatives participating in the same group who have had very similar experiences [17].

A pilot study by Hoffman et al. [25], with pre-, post-, and 6-month follow-up of one group, suggests that this program promotes significant reductions in grief and burden and a significant increase in mastery. A replication and extension study of FC by Hoffman et al. [17], with a pre-, post-, and 3-month follow-up of one group, shows improvements in well-being variables and depression. Another descriptive mixed study (qualitative and quantitative data) with two groups (family members with 
and without clinically relevant symptoms) showed that the subgroup with clinically relevant symptoms had a significant decrease in depression and anxiety symptoms at follow-up, and women showed a decrease in both anxiety and depression symptoms before and after the intervention [29]. Flynn et al. [24] found similar results in a non-randomized controlled study (pre-, post-, 3-, 12-, or 19-month follow-up) that compared FC with a psychoeducation group. Finally, in a non-randomized comparison study with pre-, post-, and 6-month followup assessments, participants who received FC reported fewer mental health difficulties, a lower perceived burden of caring, and higher overall family functioning [30].

Therefore, considerable progress has been made in this line of work, which had not previously been considered. However, it would be desirable to advance in this direction by comparing FC to active treatments in larger samples and, if possible, examine the impact of the treatment on both family members and patients. Another important issue is the dissemination of FC to other cultural contexts. This study will provide the first efficacy data on the comparison of FC with an active treatment condition in a randomized controlled trial. Another contribution of this study is the measurement of the evolution of the family climate in relation to the improvements of both relatives and patients. Finally, this is the first study on FC carried out in a Spanish-speaking population.

This study has several objectives. First, we aim to test the efficacy of FC for relatives of patients with BDP in an RCT with a sample of participants from specialized care in Spain, compared to Treatment as Usual (TAU), that is, an active treatment condition. Second, we will study the feasibility and acceptance of this intervention protocol in family members of patients with BPD. Third, we intend to study whether changes in family members' disease burden and clinical symptoms are related to the improvements observed in patients with BPD. Fourth, we aim to study whether the changes that may occur in relatives with regard to disease burden and clinical symptoms are related to the family climate. Finally, we will study the perceptions and opinions of families and patients about both intervention protocols.

We hypothesize that: a) both interventions will result in significant reductions in distress and burden and improvements in overall family functioning at post-treatment, and these results will be maintained at the 6-month follow-up; b) the FC program will significantly outperform the TAU intervention on measures of subjective burden, validation skills, family functioning, and quality of life; c) both protocols will be well accepted, but FC will be rated significantly higher by the participants; d) the improvement that may occur in the family members with regard to disease burden and clinical symptoms will have a positive influence on the family climate; e) in an exploratory way, given the lack of specific data in the literature, we hypothesize that the changes observed in the relatives will be related to the clinical evolution of the patients. In this article, we present the study protocol.

\section{Methods \\ Study design}

We will conduct a two-armed randomized controlled trial (RCT). Participants will be randomly assigned to one of two conditions: Family Connections (FC) or Treatment As Usual (TAU). Block randomization will be carried out among the three clinical centers, considering that if a patient has more than one family member who attends the group, they will be randomized together to be included in the same condition. Measures will be taken before starting the intervention, after the intervention, and at the 6-month follow-up to determine whether improvements after the intervention are maintained in the long term. The study flowchart appears in Fig. 1. We will follow the CONSORT statement (Consolidated Standards of Reporting Trials, http://www.consort-statement.org) [31, 32] and the SPIRIT guidelines (Standard Protocol Items: Recommendations for Interventional Trials) $[33,34]$.

\section{Sample size}

To determine the sample size, the effect sizes found in the literature on the subject have been considered. The controlled study by Grenyer et al. [35], which tested a group psychoeducation protocol for caregivers of people with BPD, reported medium to large effect sizes (dyadic adjustment, $d=.78$; family empowerment, $d=1.4$ ). In addition, on measures of burden, Grenyer [35] reported significant improvements between post assessment and the 12-month follow-up, with medium effect sizes (Burden Assessment Scale, $d=.45$ ). These effects are consistent with the literature on psychological treatments for other psychiatric disorders, such as the meta-analysis of psychological interventions for caregivers of people with bipolar disorders (Burden, $g=-.80$ ) [36].

Taking these data into account, in the present study, adopting a conservative approach, an effect size of 0.60 is expected because our design includes two experimental conditions. Considering an alpha of 0.05 and a statistical power of 0.80 on a 2-tailed $t$ test, the total sample size needed to reach an effect size of 0.60 for burden is 90 participants (45 participants per experimental condition). To control the maximum possible loss of participants during treatment, based on the literature about programs for family members of patients with BPD, a $29 \%$ dropout rate is expected $[20,23-25,27]$. Thus, the required sample size should be a total of 116 participants (58 participants 


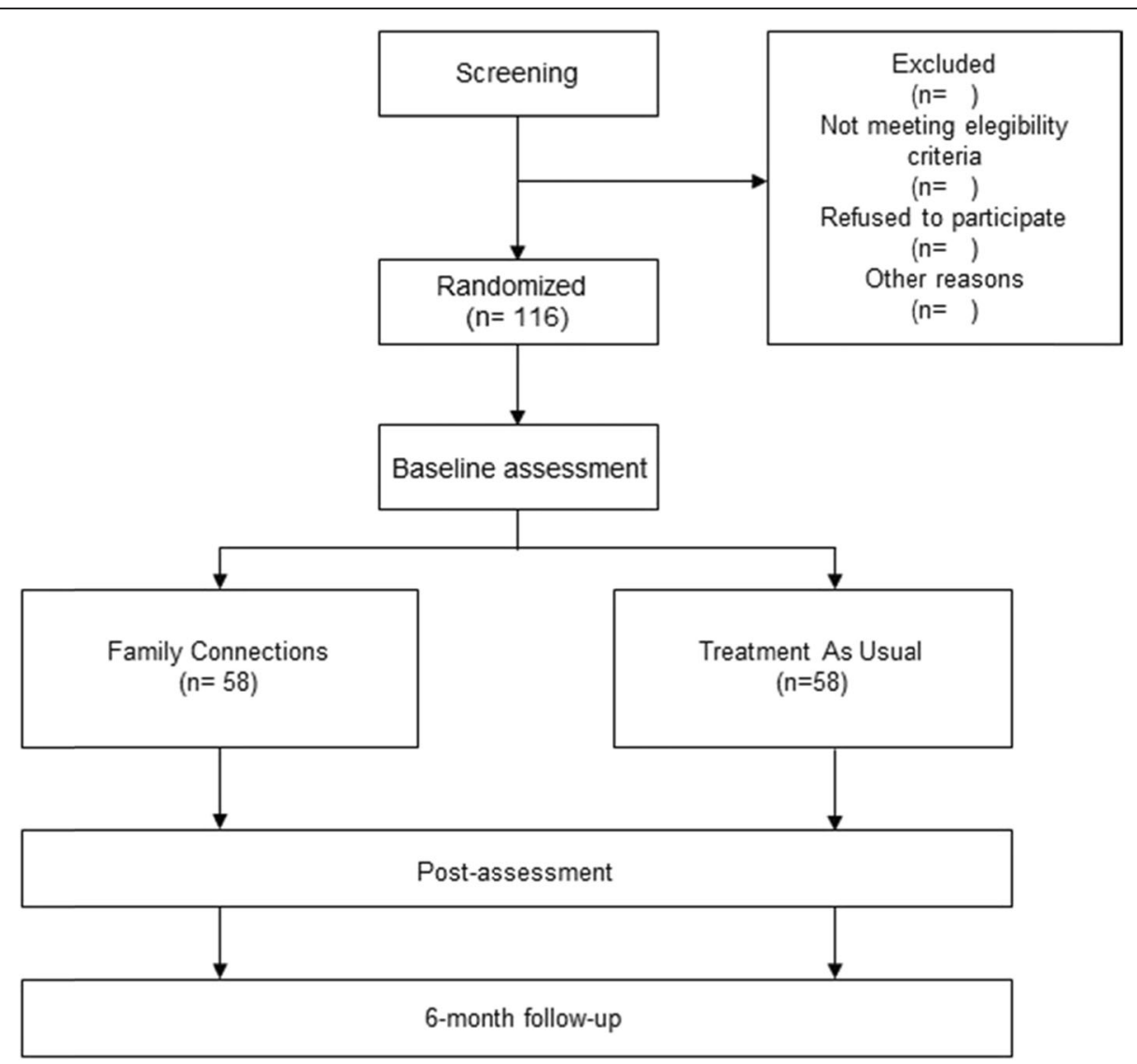

Fig. 1 Flowchart of the study

per group). These calculations were made with the software program G*Power 3.1 [37].

\section{Study population, recruitment, and eligibility criteria}

The sample will consist of relatives of people with BPD. Recruitment will be carried out among relatives of patients treated at clinical centers specializing in the treatment of BPD in the Valencian region. Inclusion criteria will include the following: a) being 18 years old or more; b) having a family member diagnosed with BPD; c) ability to understand and read Spanish; and d) providing written informed consent. Participants will be recruited by clinicians working in these clinical centers in three Spanish cities (Castellón, Valencia, and Alicante), until the required sample is complete. Clinicians will offer patients' families the opportunity to participate in the study and, after explaining it, obtain their informed consent. All the psychologists who participate in this research will have at least a master's degree in Clinical Psychology and specialized FC training.

A psychologist will contact the participant to determine his/her inclusion in the study. At that time, the researcher will collect the baseline data and determine whether the inclusion criteria are met (see Table 1).
Then the psychologist will contact a person outside the research group who will perform the individual randomization and inform the assessor of a code that corresponds to the type of treatment. This psychologist will be unaware of the characteristics of the study.

The psychologist will ensure that the participant has understood the characteristics of the study, and he/she will answer any questions the participant has. Participants will agree (or not) to participate before knowing which intervention condition they will be assigned to. The participants will also be informed that they can leave the study whenever they wish, and that in no case will there be any negative consequences for their family member who is receiving treatment at the center.

The psychologists who will participate in this study have extensive experience in implementing the DBT program for patients and will receive training in the FC program.

\section{Ethics}

The study will follow the Declaration of Helsinki Guidelines and existing guidelines in Spain and the European Union for the protection of participants in clinical trials. The Ethics Committee of the University of Valencia 
Table 1 Study measures and evaluation times

\begin{tabular}{|c|c|c|c|}
\hline Participant & Measure & Aim & Evaluation time \\
\hline \multirow[t]{7}{*}{ Caregiver } & S-D Interview & Diagnosis & $B L$ \\
\hline & BAS & Severity of burden symptoms & BL, Post-T and FU \\
\hline & FAD-GFS & Familiar Global Functioning & BL, Post-T and FU \\
\hline & DASS-21 & Depression, anxiety and stress symptoms & BL, Post-T and FU \\
\hline & FES & Family empowerment & BL, Post-T and FU \\
\hline & QLI-Sp & Quality of life & BL, Post-T and FU \\
\hline & OTSM & Treatment opinion and acceptance & PM \\
\hline \multirow[t]{5}{*}{ Patient } & FAD-GFS & Familiar Global Functioning & BL, Post-T and FU \\
\hline & DASS-21 & Depression, anxiety and stress symptoms & BL, Post-T and FU \\
\hline & DERS & Difficulties in emotional regulation & BL, Post-T and FU \\
\hline & LEAP & Emotional availability of parents & BL, Post-T and FU \\
\hline & VIRS & Validating and invalidating responses & BL, Post-T and FU \\
\hline
\end{tabular}

BL Baseline; Post-T Post-treatment; FU 6-month follow up; OTSM Opinion of Treatment Scale by Modules; S-D interview Socio-Demographic Interview; BAS Burden Assessment Scale; FAD-GFS Family Assessment Device - Global Functioning Scale; DASS-21 Depression, Anxiety and Stress Scale; DERS Difficulties in Emotion Regulation; FES Family Empowerment Scale; QLI-SP Quality Life Inventory-Spanish version; GS Grief Scale; LEAP Lum Emotional Availability of Parents; VIRS Validating and Invalidating Responses Scale

(Valencia, Spain) has approved this study. The trial was registered at clinicalstrial.gov as NCT04160871, registered the 15th of November of 2019.

Sample recruitment will be carried out by qualified clinicians. Researchers will explain the study to the participants, and they will sign the consent form as volunteer participants with the possibility of dropping out at any time. If our hypotheses are confirmed, the FC condition will be offered to participants assigned to the TAU condition after the 6-month follow-up. Special difficulties are not expected, based on the literature. If a participant drops out of the trial due to unwanted events, s/he will have the opportunity to participate the next time the treatment groups for family members are offered.

To protect information, personal data (e.g. age, sex, address, mail, phone) will be collected by the researchers participating in this study, and data will be replaced by codes. Personal data will be strictly separated from other data, and it will only be available to researchers responsible for the study, always considering and protecting the right to privacy of the participants.

\section{Interventions}

We translated the FC protocol for relatives of people with BPD into Spanish. It is one of the first programs designed to be applied directly to relatives of patients with BPD. The program is an adaptation of different Dialectical Behavior Therapy strategies, one of the most researched and empirically supported treatments for BPD people $[24,38]$. It is composed of six modules divided into 12 sessions lasting approximately $2 \mathrm{~h}$ each. The intervention protocol is structured in a caregiver handbook [25]. In the following section, the modules in each treatment program are briefly described.
The FC protocol includes components aimed primarily at reducing distress and burden and improving overall family functioning: relationship mindfulness skills, family environment skills, validation skills, and problem management skills. Furthermore, the program includes Psychoeducation about borderline personality disorder.

\section{Family connections (FC)}

This intervention program consists of six modules with two sessions each, designed to improve family attitudes and reduce family exhaustion. Each module has specific objectives and practical exercises, as well as videos with examples of people suffering from BPD and their relatives:

1. Introduction. The objective of this module is to provide information about the aims of the program, weekly format and guidelines, statement of rights, and criteria and symptoms of BPD. The central role of emotion regulation is also presented.

2. Family Education. The purpose of this module is to present treatment programs for BPD and comorbid disorders, biosocial factors related to the etiology of the disorder, the difficulties BPD provokes in the family members, and the need for help. It also shows the transactional model of the development of BPD and related disorders.

3. Relationship Mindfulness Skills. This module aims to define a validating family environment, being mindful of the relationship, emotion regulation skills, and states of mind.

4. Family Environment Skills. The aim is to understand the relationship between the individual and the family's welfare, the importance of 
maladaptive ways of thinking related to blame, and the concept of radical acceptance.

5. Validation Skills. The objective of this module is to understand what validation means and learn validation and self-validation skills. Moreover, in this module, the relatives learn how to set clear limits and achieve self-respect.

6. Problem Management Skills. This module focuses on interpersonal efficacy, defining problems and solutions, and problem management skills.

\section{Adaptation to Spanish}

The FC program has been translated into Spanish by the Puerto Rican research group directed by Dr. Domingo Marqués, and adapted to the Spanish spoken in Spain by our research team. This translation was performed by clinical experts who were familiar with both DBT [39, 40] and the FC program. The translation included the FC program manual, as well as the videos that accompany the program (they were subtitled in Spanish) and the brochures, leaflets, and handouts.

\section{Treatment as usual (TAU)}

Treatment as usual is the program routinely offered to BPD patients' relatives in the clinical centers participating in this trial. The intervention includes 12 therapeutic sessions in six modules. Each module has specific objectives and practical exercises.

1. Introduction. This module consists of an overview of the treatment and the aims of the group. Furthermore, it focuses on the definition of personality disorders, BPD and its clinical characteristics, the role of emotion regulation, and comorbid disorders.

2. Family Education. The aim of this module is to explain the diagnostic criteria for BPD, associated problems (alcohol and substance use and eating disorders), the DBT model, and the main goals of the treatment.

3. Validation Skills. The purpose of this module is to explain what validating and invalidating environments are, the consequences of an invalidating environment, and how to use validating skills.

4. Crisis Management Skills. This module aims to prevent crises by explaining how to manage anger and learning how to act in the presence of selfinjuring and suicidal behaviors. Moreover, acceptance skills are shown in this module.

5. Problem Management Skills. This module helps the relative to know how to deal with problems and set clear limits, handle conflict in everyday situations, confront unacceptable behavior, and manage emotionally charged conversations.

6. Relapse Prevention. It aims to strengthen the strategies learned throughout the program, schedule future practice, and teach the participants how to identify and cope with future high-risk situations.

\section{Measures}

Table 1 presents a summary of the measures.

\section{Caregiver measures (participants) Sociodemographic interview}

Demographic variables questionnaire: age, family constellation, sex, educational level, income, marital status, number / age of children, and psychiatric history.

\section{Primary outcomes}

Burden assessment scale (BAS) [41]

It consists of 19 items that assess the caregivers' objective and subjective burden in the past 6 months. Items are rated on a 4-point Likert scale ranging from 1 to 4 , and higher values indicate a heavier burden. Internal reliability of the scale ranges from .89 to .91 , and it shows adequate validity [42]. This scale is not validated in Spanish and it will be an objective of this work.

\section{Family assessment device - global functioning scale (FAD- GFS) [43]}

It is a self-report questionnaire consisting of 60 items related to family functioning. It is composed of seven subscales: Problem-Solving, Communication, Roles, Affective Responsiveness, Affective Involvement, Behavior Control, and General Functioning. Cronbach's alphas range from .72 to .83 for the subscales, and.92 for general functioning, and test-retest reliabilities for the FAD scales were adequate [44]. This scale is not validated in Spanish and it will be an objective of this work.

\section{Secondary outcomes}

Depression, anxiety and stress scale (DASS-21) [45]

This scale has 42 items about negative emotional symptoms. They proposed a short version with 21 items. The DASS-21 showed good factor structures. Regarding the internal consistency, Cronbach's alphas were excellent for the DASS-21 subscales: Depression $(\alpha=.94)$, Anxiety $(\alpha=.87)$, and Stress $(\alpha=.91)$ [46]. We used the Spanish version validated by Daza, Novy, Stanley and Averill [47].

\section{Family empowerment scale (FES) [48]}

It consists of 34 items divided into three subscales: family, service system, and involvement in community, which refer to three forms of empowerment: attitudes, 
knowledge, and behaviors. Items are rated on a scale from 1 to 5 , and higher scores indicate a greater sense of empowerment. The psychometric properties are the following: regarding the internal consistency of the FES subscales, the coefficients ranged from .87 to .88 , and validity and reliability were adequate. This scale is not validated in Spanish and it will be an objective of this work.

\section{Quality of life index-Spanish version (QLI-Sp) [49]}

This index consists of 10 items that assess perceived quality of life, including physical and emotional wellbeing, self-care and independent functioning, occupational and interpersonal functioning, social-emotional and community support, personal and spiritual fulfillment, and a global perception of quality of life. Higher scores indicate higher quality of life. This instrument has good psychometric properties, with a Cronbach's alpha of .89 and high test-retest reliability $(r=0.87)$.

\section{Opinion of treatment scale by modules (OTSM)}

The Opinion of Treatment Scale by Modules is an instrument developed by our research team and adapted from Borkovec and Nau [50]. It is designed to assess the participants' opinion and acceptance of the program. Furthermore, it evaluates the level of change obtained with regard to the therapeutic modules. Questions involve how logical the treatment seemed, degree of satisfaction, if they would recommend the program, if they think this program would be useful to treat their problems or others, and expectations about the program. It evaluates the six treatment modules in the two conditions. There are two subscales: one evaluates the learning of the skills taught in the module and is rated from 0 (not at all) to 10 (a lot), and the other evaluates how the module has helped the caregiver to improve several aspects, such as knowing and understanding the problem, understanding emotions, mindfulness of the relationship with their relative, acceptance, family atmosphere, and problem solving, and it is rated from 1 (not at all) to 4 (a lot). Additionally, there is an expectation question only at the end of the first module, where the participants answer the question: "In general, what expectations do you have about the program?"

\section{Patient measures}

Sociodemographic interview

Family Assessment Device - Global Functioning Scale (FAD-GFS) [43].

Depression, Anxiety and Stress Scale (DASS-21) [45].
Difficulties in emotion regulation scale - Spanish version (DERS) [51]

The authors adapted the scale to spanish and they reduced the items from 36 to 28 with five subscales: emotional lack of control, life interference, lack of emotional attention, emotional confusion, and emotional rejection. All the items have a Likert type design, with a score between 1 and 5, where 1 means "Hardly Ever" and 4 "Usually", where higher score means more difficulties. Internal consistency was excellent $(\alpha=.93)$ and good test-retest reliability $(\mathrm{pl}=.74, p<.001)$.

\section{Lum emotional availability of parents (LEAP) [52]}

It consists of 15 items that measure mothers' and fathers' emotional availability perceived by the patient. Items are rated on a 6-point Likert scale ranging from 1 (never) to 6 (always). Internal consistency was excellent in a non-clinical sample for the mother form $(\alpha=.96)$ and the father form $(\alpha=.97)$; and in a clinical sample, for the mother form $(\alpha=.92)$ and the father form $(\alpha=.93)$. This instrument has adequate test-retest reliability for the mother form $(r=.92)$ and the father form $(r=.85)$. This scale is not validated in Spanish and it will be an objective of this work.

\section{Validating and invalidating responses scale (VIRS) [53]}

The Validating and Invalidating Responses Scale is a 16item self-report that evaluates levels of validation and invalidation of caregivers' responses. This instrument has two subscales: validation and invalidation responses. These two subscales are moderately correlated. Items are rated on a 5-point Likert scale, ranging from 0 (never) to 4 (almost all the time), and higher scores indicate more perceived validation or invalidation from the caregiver who is assessed. There are no psychometric properties available on the VIRS yet. This scale is not validated in Spanish and it will be an objective of this work.

Study measures and evaluation times are summarized in Table 1.

\section{Data analyses}

In order to analyze whether there are differences between the experimental conditions before the application of the treatment, Student's t tests will be performed for the continuous variables, and chi-square tests for the categorical variables. To compare the effectiveness of the two treatment conditions, we will perform a multivariate analysis of variance for repeated measures (MANOVA) for the variables with subscales, and ANOVA for the single variables, taking the pretreatment, posttreatment and follow-up moments as within-subject factor and the treatment condition (FC vs TAU) as between-subject factor. 
Moreover, between-group changes will be computed by calculating standardized effect sizes (Cohen's d). Finally, we will perform zero-order correlations and linear regression analyses between the measures of the caregivers and the measures of the patients.

Because the trial is still going on, the state of the art in analytic methodology for RCT will be reviewed before analyzing the data, and so variations in the selection of the most appropriate analytic procedures may occur.

\section{Discussion}

FC is an intervention program for relatives of people with BPD that has been adapted to Spanish by our research team. FC was designed to train relatives of people with BPD to improve global family functioning, empowerment, resilience, validation, and mindfulness skills, and decrease grief, burden, hopelessness, and psychological symptoms [25].

The first aim of this study is to provide data from an RCT about the efficacy of this intervention protocol in a Spanish sample of participants consisting of family members of patients with BPD who are treated at clinical centers specializing in the treatment of this disorder, compared to an active condition (TAU). A second objective is to study the acceptability (expectations and opinions) of this program among the participants. Another aim is to analyze whether there are changes in relatives' burden and psychological symptoms related to the improvement observed in patients. The fourth aim consists of studying whether these changes are related to the family climate. Finally, we will examine the opinions and perceptions of relatives and patients about both intervention protocols.

The study aims to contribute to the existing literature on the efficacy and effectiveness of intervention programs for relatives of BPD patients, specifically FC. In addition, it aims to assess whether the improvements obtained in the relatives are related to those obtained by the patients themselves. Moreover, this study will help to facilitate access to this type of intervention for Spanish-speaking people, which is important due to the lack of options for many people who suffer from this problem, not only in Spain, but also in many countries in South America, in the United States, or in other countries with a significant number of Spanish-speaking citizens. The study will offer data that can be compared to those obtained in other studies carried out in Englishspeaking countries.

The data obtained in this study can be compared to results obtained in studies with DBT skills protocol programs for relatives. Several studies have found improvements in mental health patients' relatives and the relationship with their loved ones, but further research is needed. One of the aims of this study is to examine the effect of the treatment components on increasing global family functioning and decreasing burden and distress, which will mean an important change in the research and treatment of relatives of people with BPD. To our knowledge, this is the first RCT study to compare FC to an active condition (TAU) and include a 6-month follow-up.

An important aim of the study is to identify methods to improve access to $\mathrm{FC}$, as well as providing psychological support to everyone who needs it. We are living in a new era in the field of personality disorders, where BPD is given more and more attention. Researchers and clinicians are already crossing the barriers of traditional classifications and treatments, and we can now use these new protocols with significant and encouraging results. The use of the treatment in group format (a more costeffective format than individual therapy) can help to disseminate and increase the access to these family interventions.

To conclude, in this study, the effectiveness of the application of the FC program will be tested by measuring the acceptability of this program and each specific module in relatives of patients with BPD.

An important strength of this study is that it is the first RCT of FC compared to an active intervention, and it is carried out in a routine clinical care context, an ecological setting. If the hypotheses are confirmed, we expect a fast implementation of FC in these centers and other similar settings. It is also the first study carried out in a Spanish-speaking population, thus facilitating the dissemination of the program in other Spanish-speaking countries or populations.

However, this study has some limitations. We do not expect to have recruitment difficulties because our research team collaborates with different clinical centers, but even so we would have liked to increase the number of centers participating in the study. This was not possible for funding and logistic reasons. Another limitation is that we included a follow-up at 6 months. We would have liked to carry out a longterm follow-up, but due to the conditions of the centers, it is difficult to contact relatives or patients who leave treatment or are discharged.

Finally, the aim of this study is to contribute to the literature on the efficacy of the FC program for relatives of people with BPD. We hope that this study contributes to the exploration of the efficacy and acceptability of programs designed to improve global family functioning and reduce family members' burden. It will also contribute to improving our understanding of the relationships that may exist between the clinical evolution of the family members receiving the program and the evolution of the patients. If significant results are achieved, there will be an effect 
on the design and application of future family intervention programs, as a way to improve the overall functioning of the family climate and reduce the burden and distress they face. Finally, this study will allow the possible application of the program to Spanish-speaking populations in other countries.

\section{Abbreviations}

BPD: Borderline personality disorder; FC: Family connections; TAU: Treatment as usual; DBT: Dialectical behavior therapy; RCT: Randomized controlled trial; BAS: Burden assessment scale; FAD-GFS: Family assessment device-global functioning scale; DASS-21: Depression, anxiety and stress scale; FES: Family empowerment scale; QLI-Sp: Quality of life index-spanish version; OTSM: Opinion of treatment scales by modules; DERS: Difficulties in emotion regulation scale; LEAP: Lum emotional availability of parents; VIRS: Validating and invalidating responses scale; S-D: Socio-demographic; BL: Baseline; PostT: Post-treatment; FU: Follow-up; MANOVA: Multivariate analysis of variance; ANOVA: Analysis of variance

\section{Acknowledgements}

We would like to thank the participating treatment center (ITA-PREVI) and the therapists and researchers participating in this trial.

\section{Authors' contributions}

IF-F drafted the manuscript with important contributions from AG-P and VG. IF-F, in collaboration with $A G-P, V G, H M, A D-G$ and $C B$, designed the study and participated in each of its phases. AG-P, VG, CB, HM, IF-F and AD-G translated and adapted the FC program. MJ contributed to the method section. RB reviewed the content of the manuscript. All authors participated in the revision of the manuscript and have approved the final manuscript to be published.

\section{Authors' information}

IF-F is PhD student in Clinical Psychology at Universitat Jaume I in the Department of Basic and Clinical Psychology, and Psychobiology, granted by Ministry of Education, Culture and Sport (FPU) (FPU17/04210). IF-F is also member of LabPsiTec (Laboratory of Psychology and Technology) (www. labpsitec.com) at Universitat Jaume I. AG-P is full professor of Psychopathology at Universitat Jaume I in the Department of Basic and Clinical Psychology, and Psychobiology and she is also the Director of LabPsiTec. VG and HM are professors at Universitat de València, in the Department of Personality, Evaluation and Psychological Treatments, and they are members of LabPsiTec. AD-G is PhD in Clinical Psychology and member of LabPsiTec. CB is Professor Emeritus of Clinical Psychology at Universitat Jaume I in the Department of Basic and Clinical Psychology, and Psychobiology. RB is full professor of Psychopathology at Universitat de València, in the Department of Personality, Evaluation and Psychological Treatments, and she is member of LabPsiTec. MJ is the ITA-PREVI center's coordinator.

\section{Funding}

Funding for the study was provided by grants: the Ministry of Innovation Universities, Science and Digital Society (Generalitat Valenciana): I + D + I projects developed by Emerging groups. GV/2019/148 has been awarded to VG as the main investigator of the project and PhD grant has been awarded to IF-F from the Ministry of Education, Culture and Sport (FPU) (FPU17/ 04210) to produce a doctoral thesis. The study protocol was reviewed by the Generalitat Valenciana: the Ministry of Innovation, Universities, Science and Digital Society. Call for papers: Emerging research groups.

\section{Availability of data and materials}

It is not possible to share the data because the study is in progress. We are now at the stage of data recruitment.

\section{Ethics approval and consent to participate}

The study follows the guidelines of the Declaration Helsinki and existing guidelines in Spain and the European Union for the protection of patients in clinical trials. All participants interested in participating signed an informed consent form. The Ethics Committee of the University of Valencia (Valencia, Spain) has approved this study. The trial was registered at ClinicalTrial.gov as NCT04160871.

\section{Consent for publication}

"Not applicable" in this section.

\section{Competing interests}

The authors declare that they have no competing interests.

\section{Author details}

${ }^{1}$ Universitat Jaume I, Castellón, Spain. ${ }^{2}$ Universidad de Valencia, Valencia, Spain. ${ }^{3}$ ITA-PREVI (Personality Disorders Centre), Castellón, Valencia and Alicante, Spain.

Received: 9 April 2020 Accepted: 1 June 2020

Published online: 15 June 2020

\section{References}

1. Schneider B, Schnabel A, Wetterling T, Bartusch B, Weber B, Georgi K. How do personality disorders modify suicide risk? J Personal Disord. 2008;22(3): 233-45.

2. Temes CM, Frankenburg FR, Fitzmaurice GM, Zanarini MC. Deaths by suicide and other causes among patients with borderline personality disorder and personality-disordered comparison subjects over 24 years of prospective follow-up. J Clin Psychiat. 2019. https://doi.org/10.4088/jcp.18m12436.

3. Bender DS, Dolan RT, Skodol AE, Sanislow CA, Dyck IR, McGlashan TH, et al. Treatment utilization by patients with personality disorders. Am J Psychiatry. 2001;158:295-302

4. Sansone RA, Farukhi S, Wiederman MW. Utilization of primary care physicians in borderline personality. Gen Hosp Psychiatry. 2011;33:343-6.

5. Soeteman Dl, Hakkaart-van Roijen L, Verheul R, Busschbach JJ. The economic burden of personality disorders in mental health care. J Clin Psychiatry. 2008;69:259-65.

6. Meuldijk D, McCarthy A, Bourke M, Grenyer B. The value of psychological treatment for borderline personality disorder: systematic review and cost offset analysis of economic evaluations. PLoS One. 2017;12:3. https://doi.org/ 10.1371/journal.pone.0171592.

7. Amianto F, Ferrero A, Piero A, Cairo E, Rocca G, Simonelli B, et al. Supervised team management, with or without structured psychotherapy, in heavy users of a mental health service with borderline personality disorder: a twoyear follow-up preliminary randomized study. BMC Psychiatry. 2011;11:181. https://doi.org/10.1186/1471-244X-11-181.

8. Dimeff LA, Koerner K. Dialectical Behaviour Therapy in Clinical Practice. New York: Guildford: Applications across Disorders and Settings; 2007.

9. Fruzzetti A, Shenk C, Hoffman P. Family interaction and the development of borderline personality disorder: a transactional model. Dev Psychopathol. 2005:17:1007-30.

10. Bradley SJ. Relation of early maternal separation to borderline personality in children and adolescents: a pilot study. Am J Psychiat. 1979;136:424-6.

11. Links PS, Steiner M, Huxley G. The occurrence of borderline personality disorder in the families of borderline patients. J Personal Disord. 1988;2: 14-20.

12. Pope HG, Jonas JM, Hudson JI, Cohen BM, Gunderson JG. The validity of DSM-III borderline personality disorder. Arch Gen Psychiat. 1983;40:23-30.

13. Shachnow J, Clarkin J, DiPalma CD, Thurston F, Hull J, Shearin E. Biparental psychopathology and borderline personality disorder. Psychiatr. 1977;60:171-8.

14. Hoffman P, Fruzzetti A, Swenson M. Dialectical behavior therapy-family skills training. Fam Process. 1999;38:399-414.

15. Links PS. Family environment and borderline personality disorder Washington DC: American Psychiatric Press; 1990.

16. Noh S, Turner RJ. Living with psychiatric patients: implications for the mental health of family members. Soc Sci Med. 1987:25:263-72.

17. Hoffman P, Fruzzetti A. Advances in interventions for families with a relative with a personality disorder diagnosis. Curr Psychiat Rep. 2007;9:68-73.

18. Hoffma P. Family intervention: DBT family skills training. Presented at the Six International Congress on Disorders of Personality, Geneva, Switzerland, September 1999.

19. Hoffman P, Buteau E, Hooley J, Fruzzetti A, Bruce M. Family members' knowledge about borderline personality disorder: correspondence with their levels of depression, burden, distress and expressed emotion. Fam Process. 2003:42(4):469-78.

20. Rajalin M, Wickholm-Pethrus L, Hursti T, Jokinen J. Dialectical behavior therapy-based skills training for family members of suicide attempters. Arch Suicide Res. 2009;13:257-63. 
21. Dixon MD, McFarlane $W$, Lefley $H$, et al. Evidence-based practices for services to families of people with psychiatric disabilities. Psychiatr Serv. 2001;52(7):903-10.

22. Bateman A, Fonagy P. A randomized controlled trial of a mentalizationbased intervention (MBT-FACTS) for families of people with borderline personality disorder. Personal Disord. 2019;10(1):70-1.

23. Pearce J, Jovev M, Hulbert C, et al. Evaluation of a psychoeducational group intervention for family and friends of youth with borderline personality disorder. Bord Personal Disord Emot Dysregul. 2017. https://doi.org/10.1186/ s40479-017-0056-6.

24. Flynn D, Kells M, Joyce $M$, et al. Family connections versus family connections versus optimised treatment-as-usual for family members of individuals with borderline personality disorder: non-randomised controlled study. Bord Personal Disord Emot Dysregul. 2017;4:18. https://doi.org/10. 1186/s40479-017-0069-1.

25. Hoffman P, Fruzzetti A, Buteau E, et al. Family connections: a program for relatives of persons with borderline personality disorder. Fam Process. 2005: 44(2):217-25.

26. Miller ML, Skerven K. Family skills: a naturalistic pilot study of a familyoriented dialectical behavior therapy program. Couple Fam Psychol. 2017; 6(2):79-93.

27. Regalado P, Pechon C, Stoewsand C, Gagliesi P. Family relatives of persons with borderline personality disorder: a pre-experimental study of a group intervention. Vertex. 2011;22(98):245-52.

28. Wilks CR, Valenstein-Mah H, Tran H, King AM, Lungu A, Linehan MM. Dialectical behavior therapy skills for families of individuals with behavioral disorders: initial feasibility and outcomes. Cogn Behav Pract. 2017;24(2):288-95.

29. Ekdahl S, Idvall E, Perseius Kl. Family skills training in dialectical behaviour therapy: the experience of the significant others. Arch Psychiatr Nurs. 2014; 28(4):235-41.

30. Liljedahl SI, Kleindienst N, Wangby-Lundh, et al. Family Connections in different settings and intensities for underserved and geographically isolated families: a non-randomised comparison study. Bord Personal Disord Emot Dysregul. 2019;6(14). https://doi.org/10.1186/s40479-019-0111-6.

31. Moher D, Schulz KF, Altman DG. The CONSORT statement: revised recommendations for improving the quality of reports of parallel group randomized trials. J Am Podiatr Med Assoc. 2001;91(8):437-42.

32. Moher D, Hopewell S, Schulz KF, Montori V, Gotzsche PC, Devereaux PJ, et al. CONSORT 2010 explanation and elaboration: updated guidelines for reporting parallel group randomised trials. J Clin Epidemiol. 2010;63(8):e1-37.

33. Chan A-W, Tetzlaff JM, Altman DG, Laupacis A, Gotzsche PC, Krleza-Jeric K, et al. SPIRIT 2013 statement: defining standard protocol ítems for clinical trials. Ann Intern Med. 2013;158(3):200.

34. Chan A-W, Tetzlaff JM, Gotzsche PC, Altman DG, Mann H, Berlin JA, et al. SPIRIT 2013 explanation and elaboration: guidance for protocols of clinical trials. BMJ. 2013;346:e7586.

35. Grenyer BFS, Bailey RC, Lewis KL, Matthias M, Garretty T, Bickerton A. A randomized controlled trial of group psychoeducation for carers of persons with borderline personality disorder. J Personal Disord. 2019:33(2):214-28.

36. Baruch E, Pistrang N, Barker C. "Between a rock and a hard place": family members' experiences of supporting a relative with bipolar disorder. Soc Psych Psych Epid. 2018. https://doi.org/10.1007/s00127-018-1560-8.

37. Faul F, Erdfelder E, Lang A, Buchner A. G* power 3: a flexible statistical power analysis program for the social, behavioral, and biomedical sciences. Behav Res Methods. 2007:39:175-91.

38. Stoffers JM, Völlm BA, Rücker G, Timmer A, Huband N, Lieb K. Psychological therapies for people with borderline personality disorder. Cochrane Db Syst Rev. 2012;15(8):CD005652. https://doi.org/10.1002/14651858.

39. Linehan MM. Diagnosis and treatment of mental disorders. Cognitive-behavioral treatment of borderline personality disorder. New York: Guildford Press; 1993.

40. Linehan MM. DBT skills training manual. 2nd ed. New York: Guildford Press; 2015.

41. Horwitz A, Reinhard S. Family management of labeled mental illness in a deinstitutionalized era: an exploratory study. Soc Probl. 1992;4:111-27.

42. Reinhard SC, Gubman GD, Horwitz AV, Minsky S. Burden assessment scale for families of the seriously mentally ill. Eval Program Plann. 1994;17(3):261-9.

43. Epstein NB, Baldwin LM, Bishop DS. The McMaster family assessment device. J Marital Fam Ther. 1983:9(2):171-80.

44. Miller IW, Epstein NB, Bishop DS, Keitner Gl. The McMaster family assessment device: reliability and validity. J Marital Fam Ther. 1985;11(4): 345-56.
45. Lovibond SH, Lovibond PF. Manual for the depression anxiety stress scales. 2nd ed. Psychology Foundation: Sydney; 1995.

46. Antony MM, Bieling PJ, Cox BJ, Enns MW, Swinson RP. Psychometric properties of the 42-item and 21-item versions of the depression anxiety stress scales in clinical groups and a community sample. Psychol Assessment. 1998;10(2):176-81.

47. Daza P, Novy D, Stanley M, Averill P. The depression anxiety stress Scale-21: Spanish translation and validation with a Hispanic sample. J Psychopathol Behav Assess. 2002;24(3):195-205.

48. Koren PE, DeChillo N, Friesen BJ. Measuring empowerment in families whose children have emotional disabilities: a brief questionnaire. Rehabil Psychol. 1992;37(4):305-21.

49. Mezzich JE, Ruipérez MA, Pérez C, Yoon G, Liu J, Mahmud S. The Spanish version of the quality of life index: presentation and validation. J Nerv Ment Dis. 2000;188(5):301-5.

50. Borkovec TD, Nau SD. Credibility of analogue therapy rationales. J Behav Ther Exp Psychiatry. 1972;3(4):257-260. doi: 10.1016/0005- 7916(72)90045-6.

51. Hervás $G$, Jódar R. The Spanish version of the difficulties in emotion regulation scale. Clínica y Salud. 2008;19(2):139-56.

52. Lum JJ, Phares V. Assessing the emotional availability of parents. J Psychopathol Behav. 2005;27(3):211-26.

53. Fruzzetti AE. The validating and invalidating response scale-couples. Nevada: Available from Dialectical Behavior Therapy and Research Programme; 2007

\section{Publisher's Note}

Springer Nature remains neutral with regard to jurisdictional claims in published maps and institutional affiliations.
Ready to submit your research? Choose BMC and benefit from:

- fast, convenient online submission

- thorough peer review by experienced researchers in your field

- rapid publication on acceptance

- support for research data, including large and complex data types

- gold Open Access which fosters wider collaboration and increased citations

- maximum visibility for your research: over $100 \mathrm{M}$ website views per year

At BMC, research is always in progress.

Learn more biomedcentral.com/submissions 